\title{
A MATHEMATICAL FORMULATION OF THE GENERAL CONTINUOUS DEFORMATION PROBLEM*
}

\author{
BY \\ A. GLEYZAL \\ David Taylor Model Basin, Navy Department, Washington, D. C.
}

1. Introduction. The purpose of this paper is to formulate precisely and simply the general stress-strain problem for continuous flow or continuous deformation of continuous media such as viscous liquids or elastic or plastic solids. We shall use an embedded coordinate system which is considered to be embedded in the material so that each particle of the body carries with itself coordinates $x^{i}=\left(x^{1}, x^{2}, x^{3}\right)$ which ${ }^{1}$ vary continuously from particle to particle but which do not vary with time for a given particle as the body deforms.

The square of the arc length for an embedded element $d x^{i}=\left(d x^{1}, d x^{2}, d x^{3}\right)$ is given by a quadratic form $d s^{2}=g_{i i} d x^{i} d x^{i}$, summed ${ }^{2}$ on $i=1,2,3$ and $j=1,2,3$, where the fundamental tensor ${ }^{3} g_{i i}$ is a continuous function not only of $x^{i}$ but also of the time $t$. This changing fundamental tensor is of basic importance in the theory and will be called the deformation tensor.

In terms of the deformation tensor the following two principles may be stated:

1. Every continuous flow or continuous deformation may be described by the deformation tensor $g_{i j}\left(x^{i}, t\right)$ where $g_{i j}$ is the fundamental tensor of space referred to embedded coordinates in the body.

2. All equations relating stress and finite strain must be expressible as tensor equations relating a stress tensor $\sigma_{i j}$ and the deformation tensor $g_{i j}$ where $\sigma_{i j}$ and $g_{i j}$ are referred to the same embedded coordinates in the body.

Deformations or stress-strain laws which cannot be so expressed have an indefinite meaning since the deformation or stress-strain properties of the material would then depend upon the choice of coordinate system. The above principles immediately impart conceptual ease and clearness to the general precise stress-strain problem for continuous deformations. In many cases the writing of the complete equations for such a problem becomes mechanical.

Elementary examples to illustrate the procedures and equations are given in this paper. A tensor generalization of "natural strain" is found and Young's modulus and Poisson's ratio for "finite strain" are derived. Infinitesimal strain and strain velocity are defined for continuous deformations and related to the deformation tensor.

The general problem of stress and finite strain for elastic bodies has been considered by Murnaghan (3), Biot (4), and Seth (5). The author believes it would be of interest to translate the stress-strain relations assumed in these papers into tensor equations in

*Received March 6, 1948.

'Unless otherwise specified all superscripts or subscripts such as $i, j, k, \alpha, \beta$ take on the values 1, 2, and 3.

${ }^{2}$ An expression containing a letter which is repeated as a superscript and subscript is summed on that letter unless otherwise stated.

${ }^{3}$ The reader is referred to Eisenhart [1] ${ }^{4}$ and McConnell [2], for accounts on tensors and their application in geometrical and physical problems.

${ }^{4}$ Numbers in brackets refer to the bibliography at the end of this report. 
embedded coordinate systems. Definitions of finite strain based on geometrical quantities may also be so translated.

This use of a moving and deforming coordinate system may be said to be an intensification of the Lagrangian viewpoint, where the position and velocity of a particle referred to space coordinates are expressed as functions of embedded coordinates and the time $t$. It is closely related to the intrinsic viewpoint in geometry, where quantities such as the curvature of a surface are expressed in terms of the fundamental tensor of the surface itself without reference to the space in which the surface is immersed.

2. The general problem. Consider a body or continuous medium immersed in space. Let $\bar{x}^{\alpha}, \alpha=1,2,3$, be the coordinates of points of space referred to a fixed coordinate system. Let $x^{i}$ be a set of coordinates embedded in the body so that $x^{i}$ are the coordinates of a particle of the body. Any deformation may be uniquely described by three displacement functions:

$$
\bar{x}^{\alpha}=\bar{x}^{\alpha}\left(x^{i}, t\right)
$$

which prescribe the position $\bar{x}^{\alpha}$ of the particle $x^{i}$ at time $t$.

The square of the element of arc length in the space is given by a quadratic expression:

$$
d s^{2}=\bar{g}_{\alpha \beta} d \bar{x}^{\alpha} d \bar{x}^{\beta},
$$

where the coefficients $\bar{g}_{\alpha \beta}$ are known functions of $\bar{x}^{\alpha}$. Often Cartesian coordinates may be chosen so that $\bar{g}_{11}=\bar{g}_{22}=\bar{g}_{33}=1$ and $\bar{g}_{12}=\bar{g}_{23}=\bar{g}_{31}=0$. In the embedded coordinates

$$
d s^{2}=g_{i j} d x^{i} d x^{i}=\bar{g}_{\alpha \beta}\left(\frac{\partial \bar{x}^{\alpha}}{\partial x^{i}} d x^{i}\right)\left(\frac{\partial \bar{x}^{\beta}}{\partial x^{i}} d x^{i}\right) .
$$

Therefore $g_{i i}$, a function of $x^{i}$ and $t$, is given by

$$
g_{i j}=\bar{g}_{\alpha \beta} \frac{\partial \bar{x}^{\alpha}}{\partial x^{i}} \frac{\partial \bar{x}^{\beta}}{\partial x^{j}},
$$

so that $g_{i j}$ is expressible in terms of $\bar{g}_{\alpha \beta}$ and $\bar{x}^{\alpha}\left(x^{i}, t\right)$.

We shall make use of transformation equations such as

$$
\mu_{i}=\bar{\mu}_{\alpha} \frac{\partial \bar{x}^{\alpha}}{\partial x^{i}}, \quad \lambda_{i j}=\bar{\lambda}_{\alpha \beta} \frac{\partial \bar{x}^{\alpha}}{\partial x^{i}} \frac{\partial \bar{x}^{\beta}}{\partial x^{i}},
$$

where $\bar{\mu}_{\alpha}, \bar{\lambda}_{\alpha \beta}$ are the components of a tensor in $\bar{x}^{\alpha}$ coordinates and $\mu_{i}, \lambda_{i j}$ are com. ponents of the same tensor in $x^{i}$ coordinates. The formulas for raising or lowering indices of tensors $\mu_{i}$ or $\lambda_{i j}$ are formulas such as

$$
\mu_{i}=g^{i r} \mu_{r}, \quad \lambda_{i}^{i}=g^{i r} \lambda_{r i}, \quad \lambda_{i j}=g_{i r} \lambda_{i}^{r},
$$

where $g^{i r}$ is the "reciprocal" of the deformation tensor $g_{i j}$, that is,

$$
g^{i r} g_{r i}=\delta_{i}^{i},
$$

where $\delta_{i}^{i}$ is the Kroneker delta:

$$
\delta_{i}^{i}=1 \quad \text { if } \quad i=j, \quad \delta_{i}^{i}=0 \quad \text { if } \quad i \neq j .
$$

It may be noted that the six components $g^{i j}$ may each be written in terms of the six 
components $g_{i i}$ by the rules for finding the reciprocal of a matrix. It follows that $g^{i i}$ may also be expressed in terms of $\bar{g}_{\alpha \beta}$ and $\bar{x}^{\alpha}\left(x^{i}, t\right)$. Thus we may write

$$
g^{i j}=g^{i j}\left(\bar{g}_{\alpha \beta}, \bar{x}^{\alpha}\right) .
$$

In accordance with the principle stated in the introduction there exists for any material a stress-strain law of form

$$
\sigma_{i i}=\varphi_{i j}\left[g_{i j}\left(x^{i}, t\right), x^{i}, t\right],
$$

where $\varphi_{i j}$ is a functional $l^{5}$ of the six functions $g_{i j}\left(x^{i}, t\right)$ and may vary also with the numbers $x^{i}$ and $t$. In many cases it suffices to choose as $\varphi_{i j}$ a tensor formed from $g_{i j}$ and $g^{i i}$ by the processes of addition, multiplication, differentiation and integration. For example $\varphi_{i j}$ may be formed as the sum of such tensors as ${ }^{6}$

$$
g_{i i}, \int_{0}^{t} g_{i i} d t, \dot{g}_{i i}, \int_{0}^{t} \dot{g}_{i i} d t, \int_{0}^{t} \int_{0}^{t} \dot{g}_{i j} d t, \ddot{g}_{i i}, g^{r s} \dot{g}_{r i} \dot{g}_{s i}
$$

etc., with coefficients which are functions or functionals of invariants such as

$$
g^{i j} g_{i j}, g^{i j} \int_{0}^{t} \dot{g}_{i i} d t, g^{r s} g^{u v} \dot{g}_{r u} g_{s o},
$$

etc. In short $\varphi_{i j}$ is any covariant tensor of order two which may be formed from $g_{i j}$ and $g^{i i}$ by tensor addition or tensor multiplication and by differentiation or integration. For a given material $\varphi_{i j}$ is determined by experiment.

The equilibrium conditions may be written:

$$
\sigma_{i, i}^{i}=\varphi_{i, i}^{i}=0
$$

where

$$
\varphi_{i, k}^{i}=\frac{\partial \varphi_{i}^{i}}{\partial x^{k}}-\varphi_{r}^{i}\left\{\begin{array}{c}
r \\
j k
\end{array}\right\}+\varphi_{i}^{r}\left\{\begin{array}{c}
i \\
r k
\end{array}\right\}
$$

and

$$
\left\{\begin{array}{c}
i \\
j k
\end{array}\right\}=g^{i r}[j k, r],[j k, r]=\frac{1}{2}\left(\frac{\partial g_{i r}}{\partial x^{k}}+\frac{\partial g_{k r}}{\partial x^{j}}-\frac{\partial g_{j k}}{\partial x^{r}}\right)
$$

Equations (1) to (7) combine in a straightforward manner to form three equations in the three unknown functions $\bar{x}^{\alpha}=\bar{x}^{\alpha}\left(x^{i}, t\right)$ which may readily be written out. Solutions of these which satisfy the boundary conditions of the problems are sought. These boundary conditions may be, for example, specified stresses $\bar{\sigma}^{\alpha \beta}$ referred to space coordinates. They may become boundary conditions on the three functions by use of the equations: $\sigma_{i j}=\varphi_{i j}=\bar{\sigma}_{\alpha \beta} \partial \bar{x}^{\alpha} / \partial x^{i} \partial \bar{x}^{\beta} / \partial x^{i}$.

3. Relationship of $G_{i j}$ and infinitesimal strain. To translate Eqs. (2) into more familiar terms let us transform both the embedded and the space coordinates, in a given

${ }^{5} \mathrm{~A}$ functional is an association of a number with one or more functions.

${ }^{6} \mathrm{~A}$ dot placed over a quantity will indicate its time derivative when the $x^{i}$ are kept constant.

'It may be readily verified that the derivative or integral of a tensor with respect to time is also a tensor with regard to any transformation of coordinates from one embedded system to another embedded system. 
deformation, in such a way that both coordinate systems become Cartesian and have identical axes at the instant $t=t_{1}$. Then we may write

$$
\bar{x}^{\alpha}=x^{\alpha}+u^{\alpha} \text {, }
$$

where $u^{i}=u^{i}\left(x^{i}, t\right)$ are "displacements" and $u^{i}\left(x^{i}, t_{1}\right)=0$. The one-one component of the deformation tensor is, by Eq. (2),

$$
g_{11}=\left(1+\frac{\partial u^{1}}{\partial x^{1}}\right)^{2}+\left(\frac{\partial u^{2}}{\partial x^{1}}\right)^{2}+\left(\frac{\partial u^{3}}{\partial x^{1}}\right)^{2}
$$

and the one-two component is

$$
g_{12}=\left(1+\frac{\partial u^{1}}{\partial x^{1}}\right) \frac{\partial u^{1}}{\partial x^{2}}+\frac{\partial u^{2}}{\partial x^{1}}\left(1+\frac{\partial u^{2}}{\partial x^{2}}\right)+\frac{\partial u^{3}}{\partial x^{1}} \frac{\partial u^{3}}{\partial x^{2}},
$$

with similar expressions for the remaining components. It will be noted that these expressions are, except for a factor of two and the appearance of unity in some of the terms, identical with well known "exact" expressions for the "finite strain tensor". If $t$ is infinitesimally near to $t_{1}$ then $u^{1}$ is infinitesimal and

$$
g_{11}=1+2 \frac{\partial u^{1}}{\partial x^{1}}, \quad g_{12}=\frac{\partial u^{1}}{\partial x^{2}}+\frac{\partial u^{2}}{\partial x^{1}}
$$

with similar expressions for the remaining components. Now $\partial u^{1} / \partial x^{1}, 1 / 2\left(\partial u^{1} / \partial x^{2}+\right.$ $\left.\partial u^{2} / \partial x^{1}\right)$ etc., are components of infinitesimal strain as usually defined ${ }^{8}$ for the situation which exists at time $t=t_{1}$. The covariant components of strain velocity $v_{i i}$ may be defined to be the time derivatives of these infinitesimal strains at time $t=t_{1}$, so that

$$
\frac{1}{2} \dot{g}_{11}=v_{11}=\frac{\partial^{2} u^{1}}{\partial x^{1} \partial t}, \quad \frac{1}{2} \dot{g}_{12}=v_{12}=\frac{1}{2}\left(\frac{\partial^{2} u^{1}}{\partial x^{2} \partial t}+\frac{\partial^{2} u^{2}}{\partial x^{1} \partial t}\right), \quad \text { etc. }
$$

Alternatively, we may write

$$
\frac{1}{2} \dot{g}_{11}=v_{11}=\frac{\partial v^{1}}{\partial x^{1}}, \quad \frac{1}{2} \dot{g}_{12}=v_{12}=\frac{1}{2} \cdot\left(\frac{\partial v^{1}}{\partial x^{2}}+\frac{\partial v^{2}}{\partial x^{1}}\right), \quad \text { etc. }
$$

where $v^{i}=\partial u^{i} / \partial t\left(x_{i}=\right.$ const) is the velocity vector (in either coordinate system), at time $t=t_{1}$ for the particle $x^{i}$. In tensor form, therefore, the equations for strain velocity are

$$
\frac{1}{2} \dot{g}_{i i}=v_{i i}=\frac{1}{2}\left(v_{i, i}+v_{i, i}\right),
$$

where $v_{i, i}$ is the covariant derivative of $v_{i}$ defined by the equations

$$
v_{i, j}=\frac{\partial v_{i}}{\partial x^{i}}-v_{r}\left\{\begin{array}{l}
r \\
i j
\end{array}\right\}
$$

$\left\{{ }_{i}{ }_{i}\right\}$ being the Christoffel symbols as defined by Eqs. (7).

Since $\dot{g}_{i j}, v_{i i}$ and $d \epsilon_{i j}$ are each tensors with regard to any change of embedded coordinates the above results do not depend upon the choice of embedded coordinates and are valid at all times $t$. 
The infinitesimal strain at time $t$ for the time increment $d t$ is simply

$$
d \epsilon_{i i}=v_{i i} d t=\frac{1}{2} \dot{g}_{i i} d t=\frac{1}{2} d g_{i i} .
$$

Thus, assuming for convenience that $g_{i j}=0$ when $t=-\infty$,

$$
\int_{-\infty}^{t} d \epsilon_{i i}=\frac{1}{2} g_{i j}
$$

and we may say that one of the integrals of the infinitesimal strain tensor is one-half the deformation tensor in an embedded coordinate system.

By the principles stated in the introduction it was to be expected that the infinitesimal strain tensor $d \epsilon_{i j}$ should thus be expressible in terms of operations on $g_{i j}$ since surely the infinitesimal strain tensor is independent of any rigid motion of the body.

It is instructive to consider "deformations" for which $d \epsilon_{i j}=0$ at all times $t$. Such a condition may be considered to be the equivalent of a stress-strain law in a problem. If $d \epsilon_{i j}=0$ then $v_{i i}=1 / 2 \dot{g}_{i i}=0$ and the deformation tensor $g_{i i}$ is a function of $x^{i}$ alone and does not vary with time. Hence the arc length $d s^{2}=g_{i j} d x^{i} d x^{i}$ of an embedded element $d x^{i}$ is a function of $x^{i}$ and $d x^{i}$ alone and does not vary with time since $x^{i}$ and $d x^{i}$ do not vary with time. Thus the "deformation" consists of any rigid motion. The solutions of Eqs. (2) for the displacement $\bar{x}^{\alpha}=\bar{x}^{\alpha}\left(x^{i}, t\right)$ when $\bar{x}^{\alpha}$ denote Cartesian coordinates, are simply the general equations for transforming from one set of Cartesian coordinates to another such set.

Example 1. We solve a problem of a body under uniform hydrostatic pressure for the case of finite strains using a set of generalized elasticity laws. We choose as stressstrain law referred to embedded coordinates, the law:

$$
\sigma_{i j}=\varphi_{i j}=A \epsilon_{i j}+B \theta g_{i j},
$$

where $A$ and $B$ are "elastic constants",

$$
\epsilon_{i j}=\frac{1}{4} g_{i r} \int_{0}^{t} g^{r s} \dot{g}_{s i} d t+\frac{1}{4} g_{i r} \int_{0}^{t} g^{r s} \dot{g}_{s i} d t
$$

and

$$
\theta=\frac{1}{2} \int_{0}^{t} g^{i j} \dot{g}_{i j} d t .
$$

The tensor $\epsilon_{i j}$ as thus defined will be shown to be a generalization of "natural strain". It is easily verified that $\dot{\theta}^{\circ}=\dot{V} / V$, where $V$ is an element of volume of the body. Let us choose the space coordinate $\bar{x}^{\alpha}$ to be Cartesian, and the embedded coordinates $x^{i}$ to coincide with $\bar{x}^{\alpha}$ at $t=0$. Therefore

$$
\bar{g}_{11}=\bar{g}_{22}=\bar{g}_{33}=1, \quad \bar{g}_{12}=\bar{g}_{23}=\bar{g}_{31}=0 .
$$

As trial displacement functions we take

$$
\bar{x}^{i}=a x^{i},
$$

where

$$
a=a(t), \quad a(0)=1 .
$$


Using Eqs. (2) the deformation tensor is found to be

$$
g_{11}=g_{22}=g_{33}=a^{2}, \quad g_{12}=g_{23}=g_{31}=0 .
$$

The contravariant components of $g_{i j}$ are then found to be

$$
g^{11}=g^{22}=g^{33}=\frac{1}{a^{2}}, \quad g^{12}=g^{23}=g^{31}=0 .
$$

Equations (9) yield by direct computation

$$
\epsilon_{11}=\epsilon_{22}=\epsilon_{33}=a^{2} \log a, \quad \epsilon_{12}=\epsilon_{23}=\epsilon_{31}=0 .
$$

We note that raising one of the indices of $\epsilon_{i i}$ by the equation $\epsilon_{j}^{i}=g^{i r} \epsilon_{r i}$, we get

$$
\epsilon_{1}^{1}=\epsilon_{2}^{2}=\epsilon_{3}^{3}=\log a, \quad \epsilon_{2}^{1}=\epsilon_{3}^{2}=\epsilon_{1}^{3}=0 .
$$

It may be noted that $\epsilon_{i j}$ and $\epsilon_{i}^{i}$ are considered to be different components of the same strain tensor. It turns out for this example, that $\epsilon_{i}^{i}$ and $\sigma_{i}^{i}$ have identical values as the components of the strain tensor or stress tensor, respectively, calculated in experimental work. The quantity $\theta$ is found to be, for this problem: $\theta=\log a^{3}$. Since $\bar{x}^{i}=a x^{i}$ and $\bar{x}^{i}$ are Cartesian coordinates it follows that $a^{3}=V / V_{0}$ where $V$ is the volume of the body at time $t$ and $V_{0}$ is the volume at time $t=0$. Therefore $\theta=\log \left(V / V_{0}\right)$ or $\dot{\theta}=\dot{V} / V$.

Combining these equations we find

$$
\sigma_{i}^{i}=\varphi_{i}^{i}=A \epsilon_{i}^{i}+B \theta \delta_{i}^{i}=(A+3 B)(\log a) \delta_{i}^{i} .
$$

All components $g_{i j}$ are functions of time alone and do not depend on $x^{1}, x^{2}$, or $x^{3}$. The Christoffel symbols $[i j, k]$ and $\left\{\begin{array}{c}{ }_{i} \\ k\end{array}\right\}$ are linearly dependent on $\partial g_{i j} / \partial x^{k}$ and consequently vanish identically. It follows that $\varphi_{i, k}^{i}=\partial \varphi_{i}^{i} / \partial x^{k}$. Since the components are functions of time alone the quantities $\partial \varphi_{i}^{i} / \partial x^{k}$ vanish identically and we get $\varphi_{i, k}^{i}=0$. Thus the three equilibrium conditions $\varphi_{i, i}^{i}=0$ are surely satisfied.

The stress tensor at the surface of the body referred to Cartesian coordinates due to a hydrostatic pressure $p$ is $\bar{\sigma}_{\beta}^{\alpha}=p \delta_{\beta}^{\alpha}$. Hence the mixed components of the stress tensor referred to embedded coordinates are

$$
\sigma_{i}^{i}=p \delta_{\beta}^{\alpha} \frac{\partial x^{i}}{\partial \bar{x}^{\alpha}} \frac{\partial \bar{x}^{\beta}}{\partial x^{j}}=p \delta_{\beta}^{\alpha}\left(\frac{1}{a} \delta_{\alpha}^{i}\right)\left(a \delta_{i}^{\beta}\right)=p \delta_{i}^{i} .
$$

Thus, the boundary conditions of the problem are satisfied if we relate the parameter $a$ to the pressure $p$ by the equations

$$
\sigma_{i}^{i}=p \delta_{i}^{i}=(A+3 B)(\log a) \delta_{i}^{i} .
$$

or

$$
p=(A+3 B) \log a \text {. }
$$

Therefore,

$$
p=\frac{1}{3}(A+3 B) \log \frac{V}{V_{0}} .
$$

This relation for pressure and volume of a body is algebraically simple because of the particular choice of definition of finite strain (9) and the stress-strain law (8). Equations of more complexity are obtained with other stress-strain laws. For example 
we might have chosen $\epsilon_{i i}=1 / 2\left[g_{i j}\left(x^{i}, t\right)-g_{i j}\left(x^{i}, 0\right)\right]$ instead of Eqs. (9). In this case

$$
\epsilon_{11}=\epsilon_{22}=\epsilon_{33}=\frac{1}{2}\left(a^{2}-1\right), \quad \epsilon_{12}=\epsilon_{23}=\epsilon_{31}=0
$$

and

$$
\epsilon_{1}^{1}=\epsilon_{2}^{2}=\epsilon_{3}^{3}=\frac{1}{2}\left(1-\frac{1}{a^{2}}\right), \quad \epsilon_{2}^{1}=\epsilon_{3}^{2}=\epsilon_{1}^{3}=0 .
$$

The invariant $\theta$ is

$$
\theta=\epsilon_{i}^{i}=\frac{3}{2}\left(1-\frac{1}{a^{2}}\right)
$$

The equilibrium conditions $\varphi_{i, i}^{i}=0$ are again satisfied identically. At the surface of the material we have

$$
\sigma_{i}^{i}=A \epsilon_{i}^{i}+B \theta \delta_{i}^{i}=p \delta_{i}^{i}
$$

Therefore,

$$
\frac{1}{2} A\left(1-\frac{1}{a^{2}}\right)+\frac{3}{2} B\left(1-\frac{1}{a^{2}}\right)=p
$$

or, since $a^{3}=V / V_{0}$,

$$
p=\frac{1}{2}(A+3 B)\left[1-\left(\frac{V}{V_{0}}\right)^{-2 / 3} \cdot\right] .
$$

Example 2. We now apply the method to the tensile test where the stress is given by $\sigma=\sigma(t), \sigma(0)=0$. This stress is applied, let us say, in a direction parallel to the $\bar{x}^{1}$-axis of a set of Cartesian coordinates $\bar{x}^{1}, \bar{x}^{2}, \bar{x}^{3}$. Let us write for the displacement:

$$
\bar{x}^{1}=a x^{1}, \quad \bar{x}^{2}=b x^{2}, \quad \bar{x}^{3}=b x^{3},
$$

where $x^{1}, x^{2}, x^{3}$ are embedded coordinates and $a=a(t), b=b(t)$ with $a(0)=1, b(0)=1$. Then,

$$
d s^{2}=a^{2}\left(d x^{1}\right)^{2}+b^{2}\left(d x^{2}\right)^{2}+b^{2}\left(d x^{3}\right)^{2}
$$

and by inspection the deformation tensor is

$$
g_{11}=a^{2}, \quad g_{22}=g_{33}=b^{2}, \quad g_{12}=g_{23}=g_{31}=0 .
$$

The reciprocal matrix $g^{i j}$ of $g_{i j}$ has components

$$
g^{11}=\frac{1}{a^{2}}, \quad g^{22}=g^{33}=\frac{1}{b^{2}}, \quad g^{12}=g^{23}=g^{31}=0 .
$$

As stress-strain laws we choose again Eqs. (8), (9), and (10). The finite strains for this case are then:

$$
\epsilon_{11}=a^{2} \log a, \quad \epsilon_{22}=\epsilon_{33}=b^{2} \log b, \quad \epsilon_{12}=\epsilon_{23}=\epsilon_{31}=0
$$

or

$$
\epsilon_{1}^{1}=\log a, \quad \epsilon_{2}^{2}=\epsilon_{3}^{3}=\log b, \quad \epsilon_{2}^{1}=\epsilon_{3}^{2}=\epsilon_{1}^{3}=0 .
$$


Also,

$$
\theta=\frac{1}{2} \int_{0}^{t} g^{i j} g_{i i} d t=\log a b^{2} .
$$

Therefore

$$
\begin{gathered}
\varphi_{1}^{1}=A \log a+B \log a b^{2}, \quad \varphi_{2}^{2}=\varphi_{3}^{3}=A \log b+B \log a b^{2}, \\
\varphi_{2}^{1}=\varphi_{3}^{2}=\varphi_{1}^{3}=0 .
\end{gathered}
$$

All deformation components $g_{i i}$ are functions of time alone and do not depend on $x^{1}, x^{2}$, or $x^{3}$. Hence by using the same reasoning as in Example 1 it is found that the equilibrium conditions $\varphi_{i, i}^{i}=0$ are satisfied. The stresses on the boundary of the specimen referred to Cartesian space coordinates are

$$
\bar{\sigma}_{1}^{1}=\sigma, \quad \bar{\sigma}_{2}^{2}=\bar{\sigma}_{3}^{3}=\bar{\sigma}_{2}^{1}=\bar{\sigma}_{3}^{2}=\bar{\sigma}_{1}^{3}=0 .
$$

Using the transformation laws

$$
\sigma_{i}^{i}=\bar{\sigma}_{\beta}^{\alpha} \frac{\partial x^{i}}{\partial \bar{x}^{\alpha}} \frac{\partial \bar{x}^{\beta}}{\partial x^{i}},
$$

we find

$$
\sigma_{1}^{1}=\sigma, \quad \sigma_{2}^{2}=\sigma_{3}^{3}=\sigma_{2}^{1}=\sigma_{3}^{2}=\sigma_{1}^{3}=0
$$

as the mixed components of the stress tensor on the boundary of the specimen referred to the embedded coordinates. Combining these results we find

$$
\begin{aligned}
& \sigma=A \log a+B \log a b^{2}, \\
& 0=A \log b+B \log a b^{2} .
\end{aligned}
$$

Solving for $\log b$ by means of the second of these equations there results

$$
\log b=-\frac{B}{A+2 B} \log a .
$$

Therefore

$$
\sigma=A \frac{1+3 B / A}{1+2 B / A} \log a .
$$

These equations are of the form of the elasticity laws for infinitesimal strains in a tensile test if we substitute for infinitesimal strains, the "finite strains" $\epsilon_{1}^{1}=\log a, \epsilon_{2}^{2}=\epsilon_{3}^{3}=$ $\log b$. Comparing coefficients we are led to write two possible definitions of a generalized Poisson's ratio $\nu$. Either

$$
1+\nu=\left(1+\frac{3 B}{A}\right) \div\left(1+\frac{2 B}{A}\right)
$$

or

$$
\nu=\frac{B}{A+2 B} .
$$

These two definitions, it conveniently turns out, are equivalent. Hence, a generalized Young's modulus is found to be 


$$
E=A(A+3 B) \div(A+2 B) \text {. }
$$

It is furthermore seen that the finite strains $\epsilon_{1}^{1}, \epsilon_{2}^{2}, \epsilon_{3}^{3}$, as defined by (9) are in the case of a tensile test the quantities called natural strains.

The author has attempted to obtain the exact solution of the problem of the hollow cylinder under internal pressure or pure torsion and of a parallelepiped under pure shear by the general method given here. The precise conditions on the three displacement functions are readily obtained in each case for a number of stress-strain laws but their solutions require lengthy computations. The author hopes that they may be carried out in future work in this field.

It is believed that the concepts given here may be developed further to give a general theory of continuous deformation in which the notion of energy is fundamental. The author hopes to pursue these and other questions in a later paper.

\section{REFERENCES}

[1] L. P. Eisenhart, Riemannian geometry, Princeton University Press, 1926.

[2] A. J. McConnell, Applications of the absolute differential calculus, Blackie and Son Limited, 1943.

[3] F. D. Murnaghan, The compressibility of solids under extreme pressures, Kármán Anniversary Volume, 1941, p. 121.

[4] M. A. Biot, Elastizitätstheorie zweiter Ordnung mit Anwendungen, Zeitschr. angew. Math. Mech. 20,89 (1940).

[5] B. R. Seth, Finite strain in elastic problems, Phil. Trans. Roy. Soc. (A) 234, 231 (1935). 